\title{
The gas-dust transition region in young stellar objects: a sub-milli- arcsecond view through the eyes of CHARA
}

A. Tannirkulam, J. D. Monnier, R. Millan-Gabet, T. J. Harries, E. Pedretti, et al.

A. Tannirkulam, J. D. Monnier, R. Millan-Gabet, T. J. Harries, E. Pedretti, Z. Zhu, T. A. ten Brummelaar, "The gas-dust transition region in young stellar objects: a sub-milli-arcsecond view through the eyes of CHARA," Proc. SPIE 7013, Optical and Infrared Interferometry, 70130 (28 July 2008); doi: $10.1117 / 12.788512$

EDIE Event: SPIE Astronomical Telescopes + Instrumentation, 2008, Marseille, France 


\title{
The Gas-Dust Transition Region in Young Stellar Objects: A sub-milli-arcsecond view through the eyes of CHARA
}

\author{
A. Tannirkulam ${ }^{a}$, J. D. Monnier ${ }^{a}$, R. Millan-Gabet ${ }^{b}$, T. J. Harries ${ }^{c}$, E. Pedretti ${ }^{d}$, Z. Zhu ${ }^{a}$, \\ T. A. ten Brummelaar ${ }^{e}$ \\ ${ }^{a}$ University of Michigan, Astronomy Dept, 500 Church Street, 1017 Dennison Bldg, \\ Ann Arbor, MI 48109-1042, USA \\ ${ }^{b}$ Michelson Science Center, Pasadena, CA, USA \\ ${ }^{c}$ University of Exeter, School of Physics, Stocker Road, Exeter, EX4 4QL, UK \\ ${ }^{d}$ University of St. Andrews, Scotland, UK \\ ${ }^{e}$ CHARA, Georgia State University, Atlanta, GA, USA
}

\begin{abstract}
Using the sub-milli-arcsecond resolution of the CHARA interferometer array and combining light with the 2telescope combiner CHARA Classic, we have detected strong near-infrared (NIR) emission interior to the dustsublimation radius of Herbig Ae stars MWC275 and AB Aur. The large contribution of this emission component, which we argue to be hot gas, to the total NIR spectral energy distribution (SED) is not predicted by current models of the dust evaporation front, indicating that the NIR disk is more complicated than expected. Furthermore, we demonstrate that the structure of the evaporation front in MWC275 is time variable, making single epoch, large uv coverage observations critical to decoding front geometry. With the commissioning of CHARA Michigan Phase Tracker in the summer of 2008, the Michigan Infrared Combiner (a 6 telescope combiner at CHARA) will become an ideal instrument for studying the evaporation front, achieving the required sensitivities to begin the first "true" interferometric imaging of the gas-dust transition region in young stellar objects (YSOs).

Here, we summarize results on the evaporation front structure obtained with CHARA Classic and describe future prospects with CHARA MIRC in elucidating morphology of the gas-dust transition region.
\end{abstract}

Keywords: Stars: Planetary Systems: Protoplanetary Disks, Stars: Pre-Main-Sequence, Techniques: Interferometric, Radiative Transfer

\section{INTRODUCTION}

With the first-ever near-infrared interferometric observations of Young Stellar Objects (YSOs) at baselines $>200 \mathrm{~m},{ }^{1}$ we have probed the inner disk of MWC275 and AB Aur at sub-milli-arcsecond resolution. This resolution corresponds to a physical scale of $\sim 0.2 \mathrm{AU}$. On these scales, close to the central star, YSOs host fascinating astrophysical phenomena like disk winds, jets, and dust evaporation and thermal annealing.

Our targets $\mathrm{MWC} 275\left(\mathrm{~A} 1 \mathrm{e}, \mathrm{d}=122 \mathrm{pc}, \mathrm{V}=6.86, \mathrm{~K}=4.59, \mathrm{M}_{*}=2.3 \mathrm{M}_{\odot}\right)$ and $\mathrm{AB}$ Aur (A0pe, $\mathrm{d}=144 \mathrm{pc}, \mathrm{V}=7.01$, $\left.\mathrm{K}=4.37, \mathrm{M}_{*}=2.4 \mathrm{M}_{\odot}\right)$ are pre-main-sequence stars of intermediate mass known as Herbig Ae stars. This class of stars shows strong excess in near-infrared (NIR) flux ${ }^{2}$ over the stellar photospheric emission. Theories in the literature $^{3-5}$ explain this excess solely on the basis of a "puffed up inner dust rim". In these "rim" theories, dust sublimation terminates the circumstellar dust disk close to the star (the truncation radius depends on the luminosity of the central star and dust properties) creating an optically-thin cavity ${ }^{6-8}$ around the star. The outer edge of the cavity forms a dusty rim that captures stellar photons, re-radiating bulk of the energy in the NIR. The rim idea seems to broadly explain the "Size-Luminosity" correlation observed in many YSOs. ${ }^{9-11}$

However, recent results have begun to question the adequacy of models in which the entire NIR excess arises solely from dust rims. In some YSOs, NIR emission from the the dust rim alone is insufficient to explain the spectral energy distribution, ${ }^{12}$ while for others gas emission has to be included inside the dust evaporation radius to explain NIR visibilities. ${ }^{13-15}$ Due to the lack of sufficient spatial resolution, some of these conclusions are tentative and based on indirect arguments with multiple interpretations.

Send correspondence to A. Tannirkulam, E-mail: atannirk@umich.edu

Optical and Infrared Interferometry, edited by Markus Schöller, William C. Danchi, Françoise Delplancke Proc. of SPIE Vol. 7013, 70130U, (2008) - 0277-786X/08/\$18 - doi: 10.1117/12.788512 


\section{OBSERVATIONS}

To unambiguously constrain NIR structure in YSOs, we began a series of observations with the CHARA Array using the "CHARA Classic" beam combiner ${ }^{16}$. MWC275 and AB Aur were observed at K band (central wavelength of 2.13 microns) at a variety of orientations in 8 runs between June 2004 and June 2007 (see Refs. 1,17). The longest-baseline observation for MWC275 was $325 \mathrm{~m}$ (resolution * of 0.67 milli-arcseconds) and $320 \mathrm{~m}$ (resolution of 0.68 milli-arcseconds) for AB Aur. The data were reduced using standard CHARA reduction software $^{16}$ and results were cross checked with an independent code developed at University of Michigan.

Even though our targets are 2 magnitudes brighter than the sensitivity limit of CHARA, we realized early on that the low expected visibilities (see Fig 1a) at baselines $>100 \mathrm{~m}$ (based on fits to short baseline data) would make our experiment hard. In order to maximize the chances of observing fringes, we adopted the following strategy - 1) Most of the science observations were done at a dither rate of $250 \mathrm{~Hz}$. For a star with K magnitude of 4.5 (with calibrated visibility $<0.3$ ), the $250 \mathrm{~Hz}$ dither rate seems to optimize the trade off between maximizing photon counts and maintaining photon phase. 2) To locate weak fringes on targets, we first obtained fringes on nearby calibrator stars (within $15^{\circ}$ from the target) and noted the optical path length equalization facility (OPLE, Ref. 16) offset at which the fringes were located. We then moved to the target and scanned a $2 \mathrm{~cm}$ region around the calibrator offset for fringes. The OPLE carts were re-positioned in small steps and at each cart location we waited for several dithers to be completed before moving the cart again. 3) The OPLE carts try to equalize path lengths for star light based on an astrometric model. The models however are not perfect and cart positions for zero OPD (optical path difference) tend to drift relative to the model. To keep track of the OPLE offsets, we moved to a calibrator again after a $2 \mathrm{~cm}$ scan on the target. Steps 2 and 3 were repeated to identify and collect data on our target fringes.

Figure 1a shows a number of low visibility data points obtained with the manual 'fringe search' procedure.These would have been skipped over by an automated scan looking for high S/N fringes.

The uv coverage on MWC275 has allowed us to detect an asymmetry in the disk ${ }^{1,17}$ as having inclination $=48^{\circ} \pm 2^{\circ}$, $\mathrm{PA}=136^{\circ} \pm 2^{\circ}$, consistent with the inclination of $51_{-9}^{+11}$ degrees, and $\mathrm{PA}$ of $139^{\circ} \pm 15^{\circ}$ determined in Wassell et al. ${ }^{18}$ Thus, in Fig 1 the visibility of MWC275 is shown as a function of "effective baseline" -

$$
B_{\text {eff }}=B_{\text {projected }} \sqrt{\cos ^{2}(\theta)+\cos ^{2}(\phi) \sin ^{2}(\theta)}
$$

where $\theta$ is the angle between the uv vector for the observation and the major axis of the disk and $\phi$ is the inclination of the disk. Under the flat disk assumption, the effective baseline correctly accounts for the change in resolution due to the disk inclination and PA. This allows us to plot the visibility measurements as a function of one coordinate which simplifies presentation and analysis.

\section{MODELING}

To confront inner-disk theory with observations, we constructed dust-only rim models for MWC275 and AB Aur and fit the models to the visibility data. All of the K-band emission in the models is assumed to come from the inner dust rim and the central star ("standard" model). The rim is in hydrostatic equilibrium and its shape is set by the pressure dependence of the dust grain evaporation temperatures. ${ }^{4,5}$ The sublimation temperature at the base of the dust rims had to be increased to $\sim 1850 \mathrm{~K}$ from the $\sim 1400 \mathrm{~K}$ typically assumed in the literature so that the model rims fit the short-baseline $(<100 \mathrm{~m})$ visibility data. As can be seen in Fig 2, a realistic treatment of dust physics produces a curved inner rim with a sharp inner edge set by dust sublimation, and an outer edge set by 'shadowing' 3 of the region immediately behind the rim.

The standard model fits the visibility data for MWC275 and AB Aur at baselines shorter than 100m (Fig 1) fairly well (a result already established in previous studies, Refs. 8,9,19). Rim radii from the model are 0.19 AU (thickness $0.04 \mathrm{AU}$ ) for MWC275 and 0.22AU (thickness 0.05 AU) for AB Aur (see Table 1 for details). However, the standard model fails glaringly at fitting the long-baseline data, predicting strong bounces in the visibility response which are not seen in the data. Models for NIR excess in Herbig Ae stars in which all of the

\footnotetext{
${ }^{*}$ Resolution is defined as $\frac{\lambda}{2 D}-\lambda$ is wavelength of observation and $\mathrm{D}$ is the interferometer baseline length.
} 
excess arises in rims will display bounces in visibility at long baselines because of the presence of sharp ring-like features with high spatial frequency components in the corresponding images.

The absence of long-baseline visibility bounce in the data can only be explained by the presence of an additional smooth emission component within the dust destruction radius that suppresses the large visibility bounces seen in a rim-only model. The smooth emission component has to be on size scales similar to the dust sublimation radius and cannot arise from a compact source like magnetospheric accretion. Fig 2 shows a standard model to which NIR emission has been added within the dust evaporation radius in an ad-hoc manner. The data cannot uniquely constrain this emission profile and we assume a simple constant surface brightness uniform-disk model. A rim + uniform-disk model in which the uniform disk (refer Table 1 for details) accounts for 50-65\% of the total K-band emission provides a good fit to the visibility (Fig 1) over the whole baseline range.

Besides the interferometry, emission inside the dust evaporation radius can also help explain the NIR flux budget of MWC275 and $\mathrm{AB}$ Aur. In the standard models, ${ }^{4}$ the total K-band emission is only a factor of 3.5 larger (Fig 1b) than the photospheric emission, whereas MWC275 and AB Aur have total K-band emission a factor of 10 larger than the stellar photospheric K-band emission. The emission inside the dust rim can supply the "missing" K-band flux in MWC275 and AB Aur. In the next section we discuss the physical origin of this emission.

\section{PHYSICAL INTERPRETATION}

As argued in Tannirkulam et al., ${ }^{1}$ the most plausible physical mechanism for the smooth emission is hot gas. The emission needed to explain the long-baseline K-band visibility data can be obtained with optically thin gas at a $\tau \sim 0.15$ in the $2000 \mathrm{~K}-3000 \mathrm{~K}^{14,20}$ temperature range. Fig 3 plots limits on the gas opacity (normalized at $\mathrm{K}$ band) such that flux from the gas component + the standard model is within $10 \%$ of the observed photometry (we have assumed that gas does not significantly alter the geometry of the dust rim). Figs 3a and 3b show a comparison between the wavelength dependence of theoretical free-free+free-bound (henceforth FF-BF) and molecular opacities respectively ${ }^{21,22}$ with our empirically derived limits from photometry. Theoretical molecular opacities display strong bands of $\mathrm{CO}$ and water vapor at $2.5 \mu \mathrm{m}$ and $5 \mu \mathrm{m}$ which have not been observed in MWC275, suggesting that if molecules were contributing much of the NIR opacity, then some of the species are being destroyed in the inner disk.

Sitko et al. ${ }^{23}$ obtained fairly dense time coverage of the MWC275 SED and observed that NIR spectrum shows variability at the $20 \%$ level. During the same period, the stellar luminosity remained fairly constant with no detectable changes in the visible flux. A variation in the NIR morphology of MWC275 was also detected in the interferometry. The NIR disk size deduced from the April-2003 Keck Interferometer data is 20\% larger than the size obtained with the CHARA data (June 2004-Aug 2006 epochs). The size from the S2W1 June2007 data also differs at the $\sim 25 \%$ level from the size determined from earlier CHARA epochs. These variations are poorly understood and could be caused by changes in size/geometry, mass accretion rate and gas content in the inner disk.

\section{DISCUSSION AND CONCLUSION}

In this work, we have shown the presence of strong NIR emission, presumably from hot gas, inside to and on similar length scales to the dust sublimation radius in Herbig Ae stars MWC275 and AB Aur. The physical processes responsible for the emission are unknown requiring high resolution NIR spectroscopy and self-consistent modeling of the gas-dust transition region to identify the gas species and the underlying mechanism responsible for the emission.

The observed K-band sizes of MWC275 and AB Aur can be reproduced by models only if the dust sublimation temperature at the base of the dust rims is increased to $\sim 1850 \mathrm{~K}$ from the experimentally measured silicate evaporation temperatures of $\sim 1400 \mathrm{~K} .{ }^{24}$ A simple treatment of the gas-dust transition region by Muzerolle et al. ${ }^{20}$ indicates that gas does not significantly modify rim geometry. In the absence of shielding of starlight by gas, the large dust sublimation temperatures indicate that the grains in the inner disks of young stars are more refractory and/or optically transparent than typically assumed in the literature. 
The commissioning of the fringe tracker ${ }^{25}$ for CHARA-MIRC ${ }^{26}$ in the summer of 2008 will open a new observational frontier. The fringe tracker will sufficiently enhance CHARA-MIRC sensitivities to combine light from 3 or more telescopes, allowing the first milli-arcsecond non-parametric imaging of YSOs in the NIR. Fringe tracking is crucial for high spatial frequency observations where the YSOs are well resolved and visibilities are extremely low (see Fig 1). The snapshot multiple-baseline coverage will provide us a powerful tool in shedding light on the infrared time variability of YSO disks.

\section{ACKNOWLEDGMENTS}

We acknowledge contributions from PJ Goldfinger, Christopher Farrington and Steve Golden. Research at the CHARA Array is supported by the National Science Foundation through grants AST 06-06958 and AST 03-52723 and by the Georgia State University through the offices of the Dean of the College of the Arts and Sciences and the Vice President for Research. This project was partially supported by NASA grant 050283 . This publication makes use of NASA's Astrophysics Data System Abstract Service. The calibrator sizes were obtained with the fBol module of getCal, a package made available by the Michelson Science Center, California Institute of Technology (http://msc.caltech.edu).

Table 1. MWC275 and AB Aur model parameters for K-band emission. ${ }^{1} 5 \%$ of the K -band emission comes from extended envelopes. $^{27}$

\begin{tabular}{cccccc} 
Star & $\begin{array}{c}\text { model } \\
\text { type }\end{array}$ & $\begin{array}{c}\text { star } \\
\text { emission } \\
(\%)\end{array}$ & $\begin{array}{c}\text { dust-rim } \\
\text { emission } \\
(\%)\end{array}$ & $\begin{array}{c}\text { gas-disk } \\
\text { emission } \\
(\%)\end{array}$ & $\begin{array}{c}\text { rim } \\
\text { inner radius } \\
(\text { AU })\end{array}$ \\
\hline MWC275 & dust rim only & 25 & 69 & - & 0.19 \\
& dust rim+gas & 10 & 29 & 56 & 0.22 \\
AB Aur & dust rim only & 22 & 73 & - & 0.21 \\
& dust rim+gas & 12 & 22 & 65 & 0.24 \\
\hline
\end{tabular}

\section{REFERENCES}

[1] A. Tannirkulam, J. D. Monnier, R. Millan-Gabet, T. J. Harries, E. Pedretti, T. A. ten Brummelaar, H. McAlister, N. Turner, J. Sturmann, \& L. Sturmann, "Strong Near-Infrared Emission Interior to the Dust Sublimation Radius of Young Stellar Objects MWC 275 and AB Aurigae," ApJ 677, L51-L54 (2008).

[2] A. Natta, T. Prusti, R. Neri, D. Wooden, V. P. Grinin \& V. Mannings, "A reconsideration of disk properties in Herbig Ae stars," A\&A 371, 186-197 (2001).

[3] C. P. Dullemond, C. Dominik \& A. Natta, "Passive Irradiated Circumstellar Disks with an Inner Hole," ApJ 560, 957-969 (2001).

[4] A. Isella \& A. Natta, "The shape of the inner rim in proto-planetary disks," $A \& \& A 438,899-907$ (2005).

[5] A. Tannirkulam, T. J. Harries \& J. D. Monnier, "The inner rim of YSO Disks: Effects of Dust Grain evolution," ApJ 661, 374-384 (2007).

[6] R. Millan-Gabet, F. P. Schloerb, W. A. Traub, F. Malbet, J. P. Berger \& J. D. Bregman, "Sub-Astronomical Unit Structure of the Near-Infrared Emission from AB Aurigae," ApJ 513, L131-L134 (1999).

[7] P. G. Tuthill, J. D. Monnier \& W. C. Danchi, "” A dusty torus around the luminous young star LkHa101," Nature 409, 1012-1014 (2001).

[8] J. A. Eisner, B. F. Lane, L. A. Hillenbrand, R. L. Akeson \& A. I. Sargent, "Resolved Inner Disks around Herbig Ae/Be Stars," ApJ 613, 1049-1071 (2004).

[9] J. D. Monnier \& R. Millan-Gabet, "On the Interferometric Sizes of Young Stellar Objects," ApJ 579, 694-698 (2002).

[10] J. D. Monnier, R. Millan-Gabet, R. Billmeier, R. L. Akeson, D. Wallace \& 28 coauthors, "The Near-Infrared Size-Luminosity Relations for Herbig Ae/Be Disks," ApJ 624, 832-840 (2005).

[11] R. Millan-Gabet, F. Malbet, R. Akeson, C. Leinert, C., J. Monnier, J. \& R. Waters in [Protostars and Planets V], B. Reipurth, D. J. . K. K., ed., Protostarsand Planets, 539-554 (2007). 
[12] R. L. Akeson, C. H. Walker, K. Wood, J. A. Eisner, E. Scire, B. Penprase, D. R. Ciardi, G. T. van Belle, B. Whitney, J. E. Bjorkman, "Observations and Modeling of the Inner Disk Region of T Tauri Stars," ApJ 622, 440-450 (2005).

[13] J. A. Eisner, E. I. Chiang, B. F. Lane \& R. L. Akeson, "Spectrally Dispersed K-Band Interferometric Observations of Herbig Ae/Be Sources: Inner Disk Temperature Profiles," ApJ 657, 347-358 (2007).

[14] Eisner, J. A., "Water vapour and hydrogen in the terrestrial-planet-forming region of a protoplanetary disk," Nature 447, 562-564 (2007).

[15] S. Kraus, T. Preibisch, \& K. Ohnaka, "Detection of an Inner Gaseous Component in a Herbig Be Star Accretion Disk: Near- and Mid-Infrared Spectrointerferometry and Radiative Transfer modeling of MWC 147," ApJ 676, 490-508 (2008).

[16] T. A. ten Brummelaar, H. A. McAlister, S. T. Ridgway, W. G. Bagnuolo Jr., N. H. Turner \& 8 coauthors, "First Results from the CHARA Array. II. A Description of the Instrument," ApJ 628, 453-465 (2005).

[17] A. Tannirkulam, J. D. Monnier, T. J. Harries, R. Millan-Gabet, Z. Zhu \& 7 coauthors, "First Results from the CHARA Array. II. A Description of the Instrument," ApJ submitted (2008).

[18] E. J. Wassell, C. A. Grady, B. Woodgate, R. A. Kimble \& F. C. Bruhweiler, "An asymmetric outflow from the Herbig Ae star HD 163296," ApJ 650, 985-997 (2006).

[19] A. Isella, L. Testi \& A. Natta "Large dust grains in the inner region of circumstellar disks," ApJ 451, 951-959 (2006).

[20] J. Muzerolle, P. D’Alessio, N. Calvet, L. Hartmann, "Magnetospheres and Disk Accretion in Herbig Ae/Be Stars," ApJ 617, 406-417 (2004).

[21] J. W. Ferguson, D. R. Alexander, F. Allard, T. Barman \& 4 co-authors, "Low-Temperature Opacities," ApJ 623, 585-596 (2005).

[22] Z. Zhu, L. Hartmann, N. Calvet, J. Hernandez, J. Muzerolle, A. Tannirkulam, "The Hot Inner Disk of FU Orionis," ApJ 669, 483-492 (2007).

[23] M. L. Sitko, W. J. Carpenter, W. J. Carpenter, R. L. Kimes, J. L. Wilde, D. K. Lynch, R. W. Russell \& 10 coauthors, "Variability of Disk Emission in Pre-Main-Sequence and Related Stars. I. HD 31648 and HD 163296: Isolated Herbig Ae Stars Driving Herbig-Haro Flows," ApJ 678, 1070-1087 (2008).

[24] J. B. Pollack, D. Hollenbach, S. Beckwith, D. P. Simonelli, T. Roush \& W. Fong, "Composition and radiative properties of grains in molecular clouds and accretion disks," ApJ 421, 615-639 (1994).

[25] D. H. Berger, J. D. Monnier, R. Millan-Gabet, T. A. ten Brummelaar, P. Muirhead, E. Pedretti, N. Thureau, "Chara michigan phase-tracker (champ): design and fabrication," in [Advances in Stellar Interferometry], J. D. Monnier M. Schller, W. C. D., ed., Proc. SPIE 6268, 62683K (2006).

[26] J. D. Monnier, M. Zhao, E. Pedretti, N. Thureau, M. Ireland \& 11 coauthors, "Imaging the Surface of Altair," Science 317, 342- (2007).

[27] J. D. Monnier, J.-P. Berger, R. Millan-Gabet, W. A. Traub, F. P. Schloerb, E. Pedretti \& 10 coauthors, "Few Skewed Disks Found in First Closure-Phase Survey of Herbig Ae/Be Stars," ApJ 647, 444-463 (2006). 

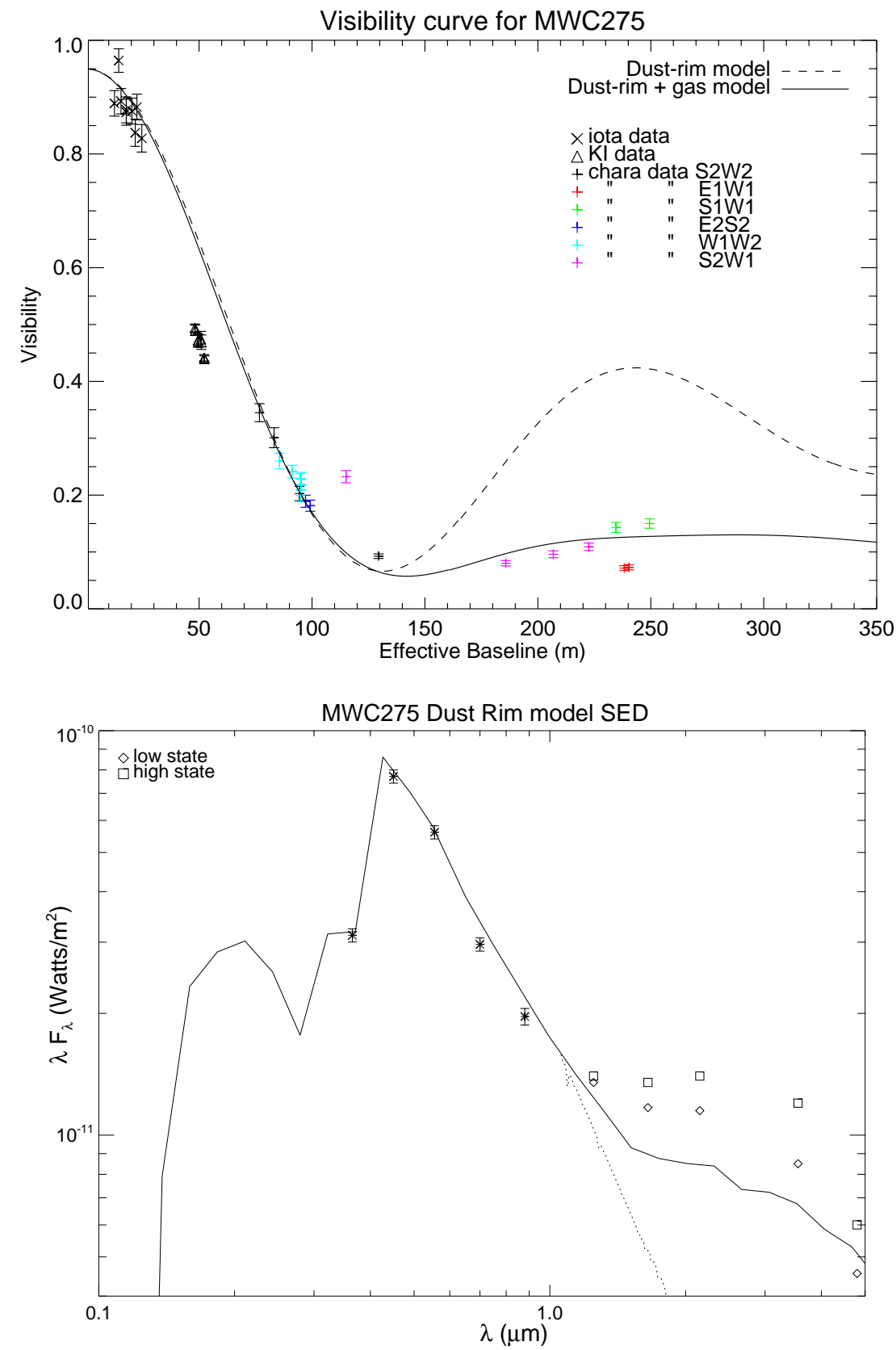

Figure 1. Visibility and SED data and model curves for MWC275. a) Top panel. MWC275 visibilities are plotted vs "effective baseline" (see section 2), which accounts for the change in resolution due to disk inclination and PA. The dotted line correspond to a "Standard" rim model tuned to fit visibility data for baselines shorter than 100m. The solid line is for a dust rim+gas model. The model parameters are listed in Table 1. b) Bottom panel. The NIR SED for MWC275. The 'stars' are photometry points from MDM. The 'squares' and 'diamonds' are high and low state measurements from Sitko et al. ${ }^{23}$ The solid line is the SED produced by the 'star + dust-rim only' model in Fig $2 \mathrm{~b}$. The dotted line is the SED of the star.

Similar results have been obtained for AB Aur as well. ${ }^{17}$

Figures reproduced from. ${ }^{1,17}$ 

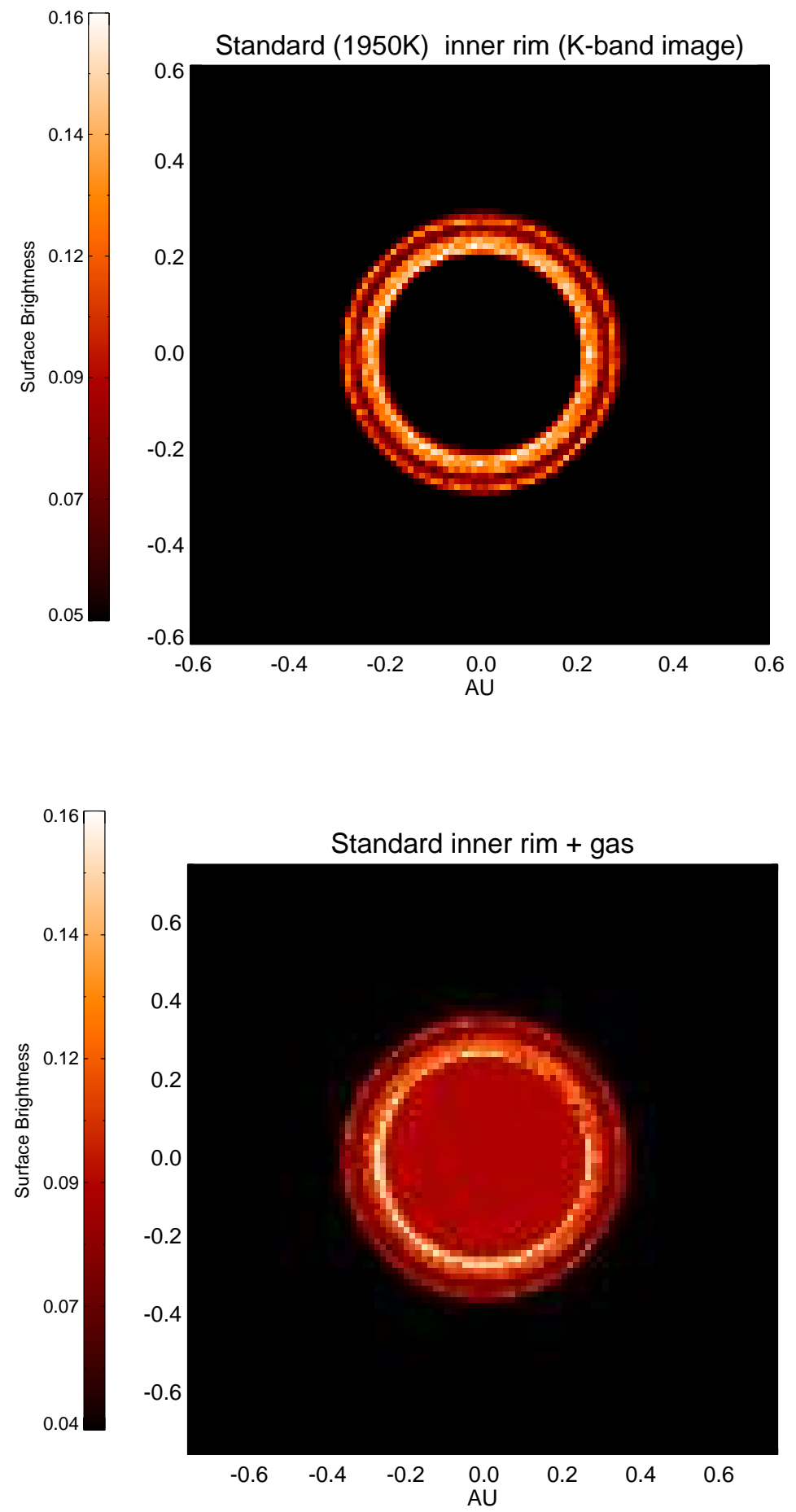

Figure 2. Illustrative models for NIR emission in Herbig Ae stars. a) Top panel. A curved rim model ${ }^{4,5}$ where the dust-sublimation temperature has power-law dependence on gas density. ${ }^{24}$ b) Bottom panel. Gas emission, modeled as a uniform disk centered on the star, has been added inside the dust rim in order to smooth out the emission profile. Figures reproduced from Tannirkulam et al. ${ }^{17}$ 

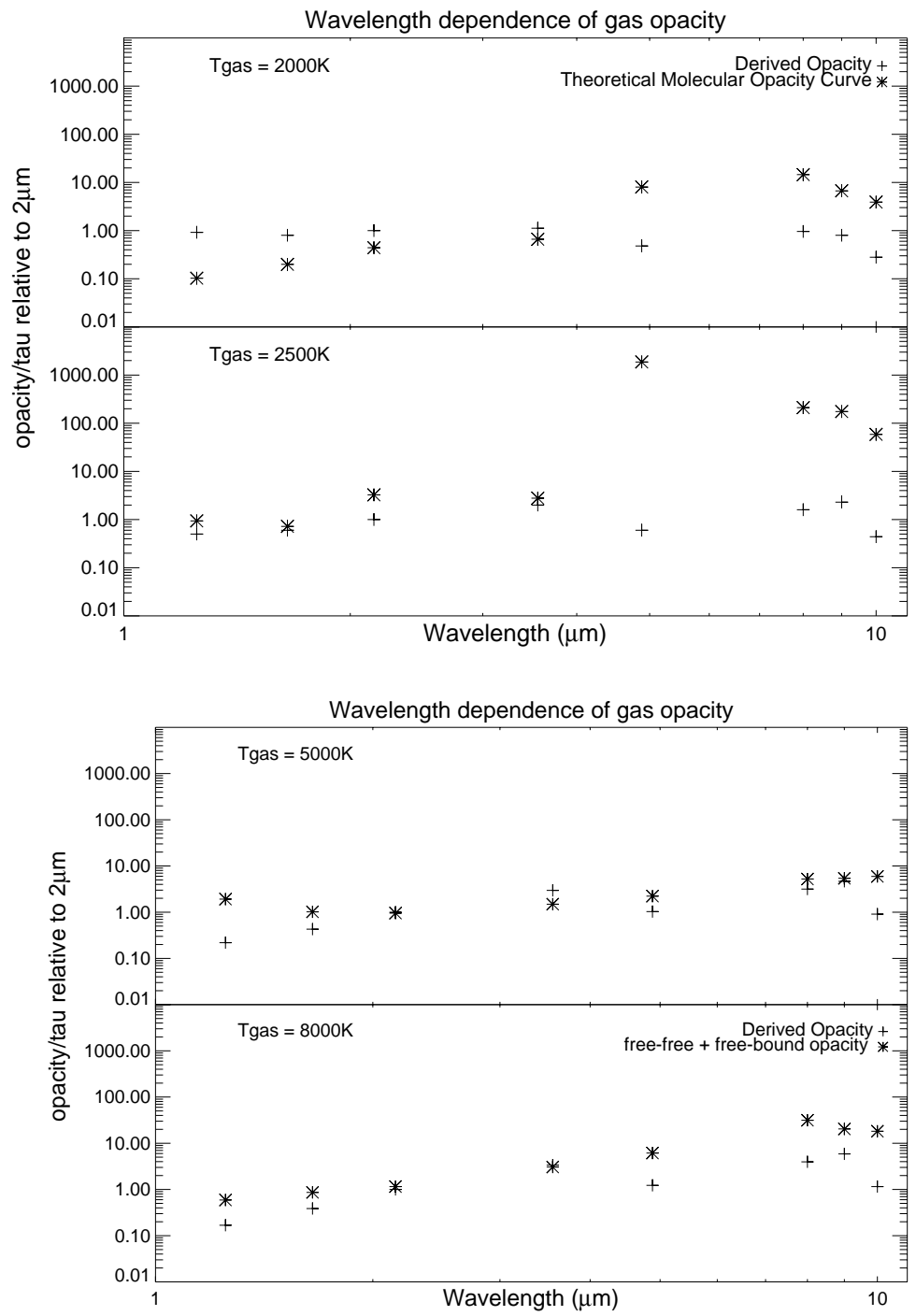

Figure 3. The plusses (+) represent gas opacities empirically derived from observed photometry and NIR disk models for MWC275 (see Fig 2). a) Top panel. The stars are theoretical molecular absorption opacities smoothed over the photometry band. ${ }^{22}$ The sharp opacity rise at $5 \mu \mathrm{m}$ is due to water vapor. b) Bottom Panel. The gas absorption opacity at infrared wavelengths is mainly from free-free and free-bound transitions of $\mathrm{H}^{-}$at $5000 \mathrm{~K}$ and hydrogen at $8000 \mathrm{~K}$. ${ }^{21,22}$ Figures reproduced from Tannirkulam et al. ${ }^{17}$ 